\title{
Preliminary insight into recognizing of mannose toward LSMT Protein: Molecular docking and DFT Study
}

\author{
Aswin Abbas ${ }^{1 *}$, Mohammad K Agusta ${ }^{2}$, Hermawan K Dipojono ${ }^{3}$, Adhitya Gadaryus Saputro ${ }^{4}$, \\ Heni Rachmawati ${ }^{5}$, Wangsa T. Ismaya ${ }^{6}$ \\ 1, 2, 3,4 Engineering Physics, Institut Teknologi Bandung, Bandung, Indonesia \\ ${ }^{5}$ School of Pharmacy, Institut Teknologi Bandung, Bandung, Indonesia \\ ${ }^{6}$ Dexa laboratories of Biomolecular Sciences, Dexa Medica, Bekasi Regency, Indonesia
}

\author{
Keywords \\ DFT \\ Interaction energy \\ LSMT \\ Mannose \\ Molecular docking
}

Received: 9 August 2018

Accepted: 5 November 2018

Published: 1 October 2018

\begin{abstract}
The present study focuses on investigating the interaction between Mannose and LSMT using molecular docking and Density Functional Theory (DFT). A novel protein like-lectin Light Subunit Mushroom Tyrosinase (LSMT) was discovered inadvertently during elucidation of the button mushroom Agaricus bisporus tyrosinase structure. The molecular docking result revealed three possible positions, of which the first resembles the sugar-binding region in the structures of its homolog (HA-33 or CNL), and the second is located in the interface region to the tyrosinase subunit. Another position is a new finding region that includes interaction with five amino acid residues. The molecule complex was modeled by truncation of five selected residues, then the atom of peptide chain freezed. In the final study, the interaction energy was analyzed using DFT showed that Threonine 91 (Thr91) has the highest role of interaction between ligand and protein. Study at this fundamental level is important because it will be used as a benchmark of interaction characteristics between LSMT and Mannose. Thus, the calculation result can be a reference in the development of LSMT application as a drug carrier protein.
\end{abstract}

(C) 2018 The Author(s). Published by TAF Publishing.

\section{INTRODUCTION}

Delivering a drug via the oral route is the most accepted and preferred way of administration because of the low cost and easiness [1]. This convenience faces problems during the drug delivering process in the body including the complexity of the digestive conditions, such as acid-base level and variation of enzymes that may cause the drug to often decompose before being absorbed. Absorption in the oral pathway requires dissolved drug molecules in the gastrointestinal tract; if the dissolution rate of the drug is significantly slower than absorption rate then dissolution is considered as the step to limit the rate of absorption $[2,3]$.

There are many ways to achieve efficient absorption of the drug through the oral route. These ways are done by adding particular ingredients to the drugs that can increase solubility, permeability, even through encapsulation of medic- inal compounds as protection from gastrointestinal conditions that inhibit the absorption of the drug. Proteins from lectin groups are widely known to increase the absorption of drugs in the intestine [4].

Lectin is used as a protein that will be the drug carrier for the oral route. The ability of lectins to recognize sugar groups on the surface of epithelial lining of the intestinal wall makes this protein highly preferred as a drug carrier. However, response from the body's immune system (immunogenicity) makes the development of the protein as a drug carrier is still lacking, so an alternative that can resemble the usability of lectins is needed. The protein proposed is LSMT, which originates from button mushroom (Agaricus bisporus). LSMT share high structural homology with Ricin-B like lectin from mushroom Clitocybe nebularis (CNL; PDB ID 3NBE) and HA-33 from Clostridium botulinum

\footnotetext{
${ }^{*}$ Corresponding author: Aswin Abbas

†email: aswien@students.itb.ac.id
} 
(HA-33, PDB ID 3AH2) [5, 6]. Moreover, the advantages of LSMT to not evoke the immune reaction in the body based on in vivo testing toward mice in Swiss Webster is appealing [7].

The functions and role of LSMT protein have not been widely studied both experimentally and computational simulation [8]. In this present work propose the preliminary study of recognition LSMT toward sugars that decorate in intestinal or on the surface of cancer cells based on computation analysis using molecular docking. Molecular docking simulation is an approach to identify the binding mode between protein and ligand at the molecular level. The molecular docking approach enables to estimate the binding structure of ligand and efficient interaction by avoiding tedious experiment in crystallography method. This approach has been successfully applied to predict the number of a particular mode of protein-ligand binding when crystallographic studies are not possible [9].

The sugar type that was selected for docking process is Mannose, based on a Surface Plasmon Resonance (SPR) analysis (data not shown). Characteristics of the interaction are measured according to hydrogen bonds between sugar molecule and amino acid residues in the protein. The level of strength or weakness of hydrogen interaction was specified by the distance between one atom of amino acid residue LSMT and one atom of ligand (sugar) [10].

For more detailed information on interactions between the individual amino acid residue of LSMT and Mannose, DFT was employed to calculate the complex (LSMT-Mannose) energy and define the point of which the atom interacts to each other after structure optimization. Study at this fundamental level is important because it will be used as a benchmark of interaction characteristics between LSMT and Mannose. Thus, the calculation result can be a reference in development of LSMT application as a drug carrier protein.

\section{COMPUTATIONAL METHODS}

The precursory study of calculation was using molecular docking to identify the region of binding possibility between Mannose and the site of LSMT. We used Autodock Vina [11] in the molecular docking technique to find binding modes and binding energy. Vina implemented local search global optimizer based on mutation and local optimization steps to define binding energy where the accepted or rejected of ligand was evaluated with a Metropolis criterion [12]. We carried out quantum mechanics method for the next stage to analyze the interaction of complex, of which its coordinate obtained from molecular docking. DFT [13] is a powerful quantum mechanics method to calculate the interaction between each amino acid residue and Mannose. Computational details both molecular docking and interaction energy will be itemized as below.

\section{A. Molecular Docking}

Preparation prior to the molecular docking simulation process is described below.

1) Preparation of protein: Protein target was obtained from the protein data bank with PDB ID: 5EHA (https://www.rcsb.org) that contains the X-Ray structure at $2.2 \dot{A}$ resolution with the $R$-value free, and $R$-value work of 0.183 and 0.162 , respectively.

2) Preparation of ligand: The mannose compound used as a ligand was from PubChem (https://bit.ly/361TTBJ). Its 3D structure was downloaded in SDV format, which was then converted in PDB file using Discovery Studio software. Prior to the docking proccess, the coordinate structure of ligand was optimized to obtain the most stable structure. Optimization used Gaussian 09 [14] software in approach DFT with B3LYP [15] hybrid functional and combined with 6-311+G (d,P) basis set.

3) Docking process: Early preparation of protein and ligand started with an operation using Auto Dock Tools (ADT) to convert PDB format to PDBQT files. The ligand was permitted to have a rotatable bond for all the atoms after the root position. In the protein molecule, all water molecules were removed and the polar hydrogen atoms were added to the refined model using ADT [16]. The grid box was set at $62,60,56$ for $x, y, z$ respectively and spacing $1.0 \dot{A}$. The grid center was set at the position 4.327, 22.503, 20.708 for $x, y$, and $z$, respectively. The grid encompasses the whole structure of ligand-protein. We posted nine poses of ligand, which generated nine binding modes of ligand toward protein based on the Gibbs free energy and Root Mean Square Deviation (RMSD) of each mode. We employed Autodock Vina [11] to dock ligand against the protein. Autodock Vina scores expressed as Gibbs free binding energies were obtained from docking calculation [17], with the most negative value of energy to show the most stable of ligand structure. All poses produced were selected and then conjugated with the protein using Discovery Studio software. The structure of the complex (ligand-protein) was saved in PDB format. Subsequently, the complex was analyzed using LigPlot [18] to find the number and type of amino acid residues that interact with the ligand. In addition, hydrogen and hydrophobic bonds was identified by LigPlot plotting. 


\section{B. Interaction Energy}

Elaboration of the interaction energy between Mannose and LSMT was done using DFT analysis. We took some amino acid residues based on molecular docking result. The coordinate of Mannose and all of selected individual residues extracted from the best binding mode of Mannose toward protein (the lowest free energy). Interaction energy Eint was obtained by equation.

$$
E_{\text {int }}=E_{\text {complex }}-E_{\text {ligand }}-E_{\text {residue }}
$$

where $E_{\text {complex }}$ is the energy of the complex between Mannose and individual amino acid residue in LSMT, then $E_{\text {ligand }}, E_{\text {residue }}$ are energy of Mannose and individual amino acid residue respectively. DFT was employed to calculate the optimized structure of complex, ligand, and amino acid residues. The structure optimization is carried out by Gaussian09 software package with B3LYP [15] exchange correlation and $6-311++G(d, p)$ basis set. The atom in peptide bonds of amino acid residue was fixed as compensation for truncation of other residues (Figure 1).

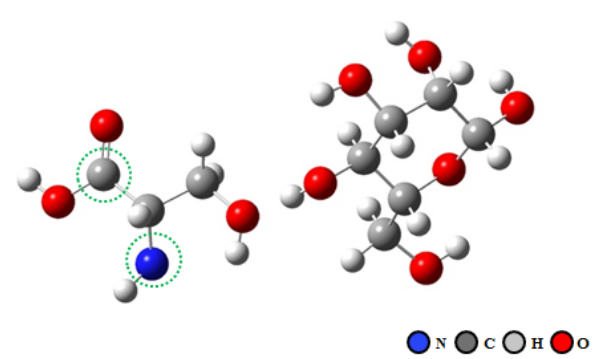

Fig. 1. Complex of Mannose (right) and one of residue (left). The green dotted is peptide bonds which was fixed and the rest of atoms was in relax

\section{RESULTS AND DISCUSSION}

\section{A. Molecular Docking}

Molecular docking process was carried out with specific amino acid residue that was used as a reference to build grid box in the protein. Selected the residues based on pre- vious docking in CNL which consist of Asn24, Ser26, Thr37, Leu38, Asp42, and Ser44 [5]. Docking result revealed that there are three regions in LSMT that can facilitate ${ }^{2}$ binding with Mannose. Several poses were docked in each region which is composed according to the binding affinity energy level (Figure 2).

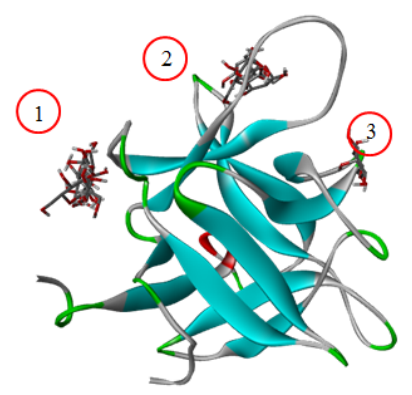

Fig. 2. Binding region between some candidates of Mannose toward LSMT

The first region consists of five candidates of Mannose, where every candidate has different binding energy values and binding mode. In addition, this region is occupied by the tyrosinase subunit in the mushroom tyrosinase tetrameric complex. The second region comprises three candidates and this site resembles the ligand-binding re- gion in HA-33 or CNL. Both region one and two is consistent with the previous docking [5]. The present result revealed a new binding site of the region 3 that contains a pose with the most favorable binding based on its lowest free energy and RMSD. 
Details of docking result are presented in Table 1. It can be seen that the most stable result occurred in mode 1 of the region 3 with energy $-5.7 \mathrm{kcal} / \mathrm{mol}$ and RMSD $\sim 0.00 \dot{A}$. Of the three regions in which the Mannose forms interaction with LSMT, each existing mode 1,2 , and 5 are selected for the most stable binding in the region $3,2,1$, respectively. There are several amino acid residues that interact with Mannose based on distances between atom of residues and Mannose. The most favorable binding of Mannose toward LSMT as shown in Figure 3.

TABLE 1 DOCKING BETWEEN MANNOSE AND LSMT

\begin{tabular}{llll}
\hline \hline Mode & Energy $(\mathrm{kcal} / \mathrm{mol})$ & RMSD $(\dot{A})$ & Region/site \\
\hline 1 & -5.7 & 0.00 & 3 \\
2 & -5.7 & 12.832 & 2 \\
3 & -5.5 & 12.474 & 2 \\
4 & -5.5 & 13.535 & 2 \\
5 & -5.5 & 25.320 & 1 \\
6 & -5.4 & 25.380 & 1 \\
7 & -5.3 & 25.753 & 1 \\
8 & -5.2 & 25.206 & 1 \\
9 & -5.1 & 26.346 & 1 \\
\hline \hline
\end{tabular}

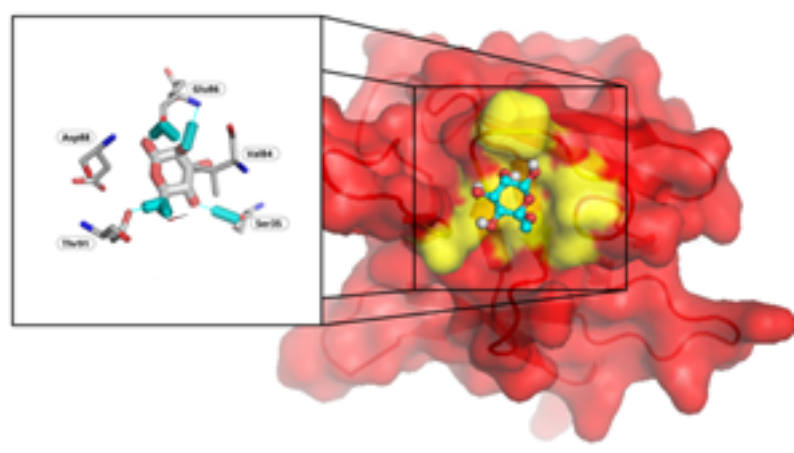

Fig. 3. The best binding mode between Mannose and LSMT based on the lowest free energy
The binding mode in every region has different types and numbers of amino acid residues interacting with Mannose. At mode 1, there are Ser35, Glu86, and Thr91 in hydrogen binding, meanwhile hydrophobic interaction consists of Val84 and Asp88. The residue Glu86 experiences the highest interaction due to its distance is closer $(2.89 \dot{A})$ than other. However, the interaction occurs between the C-2 Mannose and the carbon backbone of the amino acid, not through its side chain. Mode 2 includes five hydrogens bonding with residues Ala27, Thr28, Glu29, Asn30, and Asn34. Hydrophobic interaction encompasses Gly36 and Thr37 with the closest distance is $2.88 \dot{A}$ for hydrogen binding at Glu29. As shown in Figure 3 (right), mode 5 involves five hydrogens bonding with residues Arg15, Thr45, Asp49, Asp51 and Arg52 while hydrophobic interaction only one residue Gln48. Further study regarding the interaction energy of the complex LSMT-Mannose (or individual residue and Mannose) was focused on the bonding mode 1 (region 3).

\section{B. Interaction Energy}

Molecular docking suggested the best position and binding mode for Mannose upon binding to amino acid residues in LSMT. However, molecular mechanics, who is the principal core engine of molecular docking, did not enable to explicate binding and interaction of the atoms. Identification of atomic interaction between Mannose and LSMT (complex) employed optimization of complex structure using DFT $[13,19]$. The calculation of the interaction energy of the complex was expressed in Equation1. Each amino acid residue was calculated and the whole summation of them represents the interaction energy between Mannose and pocket LSMT (yellow region in the Figure 3 ). The interaction, however, did not distinguish the atoms that build up the side chain and the polypeptide backbone i.e. $\mathrm{N}$-(amine) and C-(carboxyl) termini, and the $\mathrm{C} \alpha$.

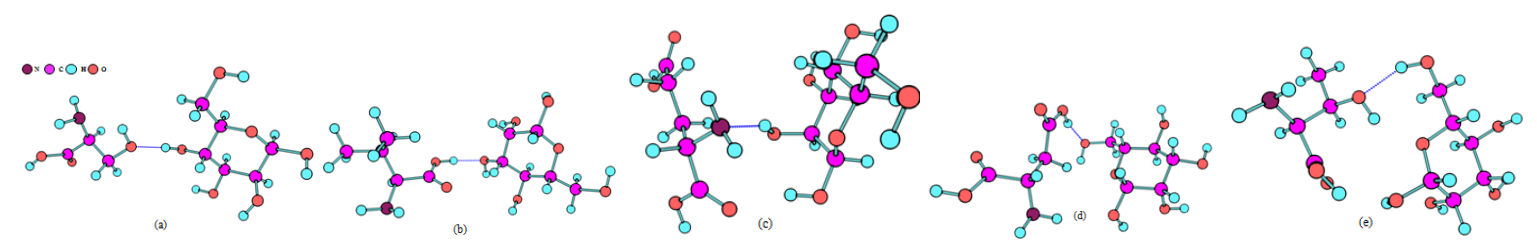

Fig. 4. Interaction of molecule between individual amino acid residue and Mannose; (a) Mannose-Ser35, (b) Mannose-Val84, (c) Mannose-Glu86, (d) Mannose-Asp88, and (e) Mannose-Thr91

The calculation result of the complex Mannose-Ser35 shows hydrogen binding by hydrogen atom of Mannose to oxygen atom of Ser35 side chain with distance $1.84 \dot{A}$ and interaction energy $-11,831 \mathrm{kcal} / \mathrm{mol}$. This binding is highly fa- vorable. However, it occurs with the C-4 of the Mannose thereby not corresponding to the sugar specificity. Subse quently, the complex Mannose-Val84 has the shortest distance of two molecules atoms $1.79 \dot{A}$ and interaction energy 
$-11.695 \mathrm{kcal} / \mathrm{mol}$. This interaction occurs between the C-3 of the Mannose and the side chain, this is not specific for Mannose as well as the methyl group of Val84 hydrophobic character is less likely to form hydrogen bond. Complex Mannose-Glu86 has interaction energy $-4.879 \mathrm{kcal} / \mathrm{mol}$ and $1.96 \dot{A}$ is the distance of those atom molecules. The interaction occurs with the $\mathrm{C}-2$ Mannose, which defines the specificity. However, the hydroxyl group of C-2 Mannose interacts with the $\mathrm{N}$ of amine, which belongs to the peptide bond. This situation is possible but also unlikely to occur. Moreover, the binding is then not specifically referring to the amino acid because any residues in the polypeptide chain are connected through this bond. Meanwhile, the interaction energy of the complex Mannose-Asp88 is $-9.021 \mathrm{kcal} / \mathrm{mol}$ with the distance $1.87 \dot{A}$. This interaction occurs between the carboxyl side chain and the hydroxyl of the Mannose C-5, thus is not specific. The last complex Mannose-Thr91 has interaction energy $-13.609 \mathrm{kcal} / \mathrm{mol}$ and distance of atom complex molecule $1.89 \dot{A}$. According to accumulation the whole energy of individual residues then obtained interaction Mannose to pocket of protein $-51.037 \mathrm{kcal} / \mathrm{mol}$ as noted that Thr91 gave the highest contribution (percent unit) of interaction energy as shown in Figure 5. This interaction occurs between the hydroxyl side chain and the Mannose $\mathrm{C}-5$, thus it is also not specific. The calculation thereby managed to dock the Mannose molecule nicely in the protein surface but unfortunately no specific interaction was observed.

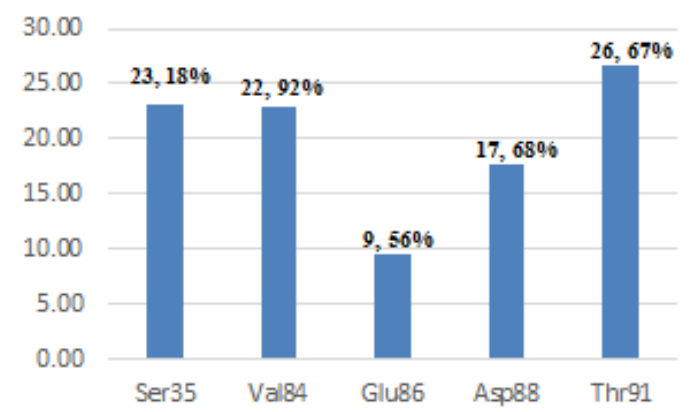

Fig. 5. Level contribution of interaction energy each individual residue toward Mannose

The interaction energies profile in Figure 5 suggests that Thr91 has the highest contribution of interaction toward
Mannose based on its lowest energy. However, Threonine (Thr) is basically amino acid with polar side chain but uncharged. In nature, Thr is often found to form a covalent bond with oxygen (glycosylation). In the case of LSMT, the protein did not form covalent bond with the Mannose. The finding of Thr91 as the amino acid with the most influential interaction appears to dissagree with the experimental result, which suggests that binding of Mannose to the amino acid residue specifically involves the hydroxyl group at the position C-2 of pyranose ring. This was demonstrated by Glu86 although the interaction occurs with the polypeptide main chain, therefore not amino acid-specific. There are factors that make discrepancy of result calculation and experiment, including truncating most amino acid in the calculation process then generate a steric effect of molecule structure. Furthermore, a calculation that involves the entire system is highly recommended to get a more accurate result.

\section{CONCLUSION}

Recognition of Mannose sugar molecule by LSMT protein has been studied. Molecular docking has been employed to find a region and the best mode binding for Mannose to the LSMT. Two of three regions resemble the site of HA-33 and CNL while one region is a new binding site. Docking and fitting results of Mannose-6-phosphate resulted in binding at a similar region but including addition of Arg77. Thus, the molecular docking study shows Mannose's potential to bind in LSMT. In order to identify which individual residue that has the highest role in interaction complex, DFT was employed as a powerful method. Thr91 was found to have the most stable energy but this finding was not in agreement with the experiment result. Thus, further calculation should be done employing the method that is more accurate that involves the entire system.

\section{ACKNOWLEDGMENT}

We are thankful to Indonesia Endowment Fund for Education (LPDP) as a provider of funding support for this paper. Also, We thank Muniba Faiza (South China University of Technology) for sharing and constructive discussions regarding molecular docking.

\section{REFERENCES}

[1] N. Jawahar, P. K. Hingarh, R. Arun, J. Selvaraj, A. Anbarasan, S. Sathianarayanan, and G. Nagaraju, "Enhanced oral bioavailability of an antipsychotic drug through nanostructured lipid carriers," International Journal of Biological Macromolecules, vol. 110, pp. 269-275, 2018. doi: https://doi.org/10.1016/j.ijbiomac.2018.01.121

[2] A. Beloqui, A. del Pozo-Rodríguez, A. Isla, A. Rodríguez-Gascón, and M. Á. Solinís, "Nanostructured lipid carriers as oral delivery systems for poorly soluble drugs," Journal of Drug Delivery Science and Technology, vol. 42, pp. 144-154, 2017. doi: https://doi.org/10.1016/j.jddst.2017.06.013 
[3] N. Mahmad, R. M. Taha, N. Rawi, and S. Mohajer, "The effects of picloram and 2,4-dichlorophenoxyacetic acid on induction of red coloured callus from celosia plumosa, an attractive ornamental plant," Journal of Applied and Physical Sciences, vol. 1, no. 1, pp. 9-12, 2015. doi: https://doi.org/10.20474/-japs1.1.2

[4] L. L. De Juan, V. G. Recio, P. J. López, T. G. Juan, M. Cordoba-Diaz, and D. Cordoba-Diaz, "Pharmaceutical applications of lectins," Journal of Drug Delivery Science and Technology, vol. 42, pp. 126-133, 2017. doi: https://doi.org/10.1016/j. jddst.2017.05.018

[5] W. Ismaya, S. Damayanti, C. Wijaya, R. Tjandrawinata, D. Retnoningrum, H. Rachmawati et al., "In silico study to develop a lectin-like protein from mushroom agaricus bisporus for pharmaceutical application," Scientia Pharmaceutica, vol. 84, no. 1, pp. 203-217, 2016.

[6] B. S. K. Bhandary, K. P. Sharmila, N. S. Kumari, V. S. Bhat, and G. Sanjeev, "Radioprotective potential of punica granatum extract and synthetic ellagic acid: A biochemical and hematological study in mice," International Journal of Applied and Physical Sciences, vol. 1, no. 3, pp. 51-54, 2015. doi: https://doi.org/10.20469/ijaps.50001-3

[7] W. T. Ismaya, A. Efthyani, X. Lai, D. S. Retnoningrum, H. Rachmawati, B. W. Dijkstra, R. R. Tjandrawinata et al., "A novel immune-tolerable and permeable lectin-like protein from mushroom agaricus bisporus," Biochemical and Biophysical Research Communications, vol. 473, no. 4, pp. 1090-1093, 2016. doi: https://doi.org/10.1016/j.bbrc.2016.04.020

[8] W. T. Ismaya, O. M. Tandrasasmita, S. Sundari, X. Lai, D. S. Retnoningrum, B. W. Dijkstra, R. R. Tjandrawinata, H. Rachmawati et al., "The light subunit of mushroom agaricus bisporus tyrosinase: Its biological characteristics and implications," International Journal of Biological Macromolecules, vol. 102, pp. 308-314, 2017. doi: https://doi.org/10.1016/ j.ijbiomac.2017.04.014

[9] S. Sokalingam, G. Munussami, J.-R. Kim, and S.-G. Lee, "Validation on the molecular docking efficiency of lipocalin family of proteins," Journal of Industrial and Engineering Chemistry, vol. 67, no. 6, pp. 293-300, 2018.

[10] J. Dannenberg, An Introduction to Hydrogen Bonding. New York, NY: ACS Publications, 1998.

[11] 0. Trott and A. J. Olson, "Auto dock Vina: Improving the speed and accuracy of docking with a new scoring function, efficient optimization, and multithreading," Journal of Computational Chemistry, vol. 31, no. 2, pp. 455-461, 2010. doi: https://doi.org/10.1002/jcc.21334

[12] L. Ivanova, J. Tammiku-Taul, Y. Sidorova, M. Saarma, and M. Karelson, “Small-molecule ligands as potential GDNF family receptor agonists," ACS Omega, vol. 3, no. 1, pp. 1022-1030, 2018. doi: https://doi.org/10.1021/acsomega.7b01932

[13] W. Kohn and L. J. Sham, "Self-consistent equations including exchange and correlation effects," Physical Review, vol. 140, no. A, pp. 11-33, 1965. doi: https://doi.org/10.1103/PhysRev.140.A1133

[14] Gaussian, "G09," 2019. [Online]. Available: https://bit.ly/2r2s6T3

[15] A. D. Becke, "Density-functional thermochemistry iii: The role of exact exchange," Journal of Chemistry and Physics, vol. 98, no. 7, p. 5648-5652, 1993. doi: https://doi.org/10.1063/1.464913

[16] D. Shaji, "Molecular docking studies of human MCT8 protein with soy isoflavones in Allan-Herndon-Dudley Syndrome (AHDS)," Journal of Pharmaceutical Analysis, vol. 8, no. 5, pp. 318-323, 2018. doi: https://doi.org/10.1016/j.jpha.2018. 07.001

[17] L. Ozalp, S. S. Erdem, B. Yüce-Dursun, Ö. Mutlu, and M. Özbil, “Computational insight into the phthalocyanine DNA binding via docking and molecular dynamics simulations," Computational Biology and Chemistry, vol. 77, pp. 87-96, 2018. doi: https://doi.org/10.1016/j.compbiolchem.2018.09.009

[18] R. A. Laskowski and M. B. Swindells, LigPlot+: Multiple Ligand Protein Interaction Diagrams for Drug Discovery. New York, NY: ACS Publications, 2011.

[19] P. Hohenberg and W. Kohn, "Inhomogeneous electron gas," Physical Review, vol. 136, no. 3, pp. 864-871, 1964. doi: https://doi.org/10.1103/PhysRev.136.B864 